\title{
Cross-identifications and astro-photometric comparisons of the ICRF with recent catalogs of quasars
}

\author{
J. Souchay, A. M. Gontier, and C. Barache
}

\author{
Observatoire de Paris, SYRTE, 61 avenue de l'observatoire, 75014 Paris, France \\ e-mail: jean.souchay@obspm.fr
}

Received 19 October 2005 / Accepted 10 January 2006

ABSTRACT

\begin{abstract}
The ICRF represents the most accurate astrometric catalog that is the primary reference frame adopted by the IAU General Assembly at Kyoto in 1997. Despite its importance, an exhaustive study of both the sky coverage and the physical properties of the extragalactic sources representing the ICRF is lacking. The object of the present paper is to extract some information concerning these sources and cross-identify them with the data gathered by Véron-Cetty \& Véron (2003, A\&A, 412, 399), which is the largest compilation of various catalogs of quasars. After this cross-identification of the sources, we carry out a study of some general features of the ICRF concerning the visual magnitudes, the redshifts, and the flux at radio wavelengths
\end{abstract}

Key words. astrometry - quasars: general - surveys

\section{Introduction}

The ICRF (International Celestial Reference Frame) was adopted as the fundamental celestial reference frame at the XXIII General Assembly of the International Astronomical Union held on August 1997 in Kyoto. It is considered as the realization of the ICRS (International Celestial Reference System), and is currently defined by the coordinates of 212 extragalactic objects obtained exclusively by the VLBI (Very Long Baseline Interferometry) technique during more than 20 years, at $S$ and $X$ bands, corresponding to the frequencies $2.3 \mathrm{GHz}$ and $8.4 \mathrm{GHz}$ respectively, that is to say to the wavelengths of $13.0 \mathrm{~cm}$ and $3.6 \mathrm{~cm}$ (Ma et al. 1998). The ICRF has advantageously replaced the FK5 optical catalog (Fricke et al. 1988) as the fundamental reference frame, which became effective on January 1st, 1988. In parallel, the Hipparcos catalog must be considered as the primary reference frame at optical wavelength (see for instance Kovalevsky 1998), designated as the Hipparcos Celestial Reference Frame or HCRF (IAU 2000).

Quasars have been known for roughly 40 years now, since the discovery of 3C 273 by Hazard, Mckey and Shimings. Moreover, in recent years a considerable effort has been made in their detection. The various updates in compiling several catalogs of quasars carried out by Véron-Cetty \& Véron (2001, 2003) are a clear indication of this improvement, when the number of sources recorded during the 11 successive releases by the above authors from 1984 to 2003 switched from roughly 2500 to 50000 sources. The introduction of a database coming from large and deep surveys as the SDSS (Sloan Digital Sky Survey) and the $2 \mathrm{dF}$ quasar catalog are at the origin of a particularly large increase in recorded quasars in the past few years.

In this paper we give an overall comparison of the sources recorded in extended versions of the ICRF and those recorded in the last Véron-Cetty \& Véron catalog (2003) in two different fields: the first one concerns the physical properties (flux at visual and radio waveslengths, redshifts, etc.); the second concerns the sky coverage. We thus show significant differences between these two catalogs. This study might be useful for constructing a primary reference frame in the future, based on a much larger number of extragalactic sources in the ICRF itself.

\section{The ICRF and its two extensions, ICRF-Ext.1 and ICRF-Ext.2}

In 1995, i.e. roughly ten years ago, a subgroup of the IAU working group on reference frames was set up with the task of constructing the ICRF as a new reference frame based on the VLBI observations of quasars. In 1997 at the IAU General Assembly at Kyoto, the IAU established the ICRS (nternational celestial reference system) and adopted the ICRF (international celestial reference frame) as the replacement of the FK5 (Fundamental Katalog 5) constructed starting from the positions of stars (Fricke et al. 1988). This replacement officially took place on January 1st, 1998. The ICRF is currently defined by the radio positions of 212 extragalactic objects, usually called "defining sources", obtained from VLBI observations consisting of 1.6 million pairs of group-delay and phase-delay-rate observations (Ma et al. 1998). The basic concept for the establishment of the ICRF is a no net rotation condition satisfied by the frame. In other words, no defining source shows any significant motion with respect to the other ones, so that the frame can be considered as rigid.

The positional accuracy of the ICRF sources is better than about 1 mas in both equatorial coordinates, with a median uncertainty of 0.4 mas on the positions. The coordinates of the "defining" sources implicitely define the position of the three rectangular axes of the ICRF. In addition to the defining sources, 294 objects called "candidate" sources were selected. These are compact sources that look stable enough to be considered in the future as defining sources, but whose positions are likely to be improved when more observations are accumulated in the future. Along with defining and candidate sources, 102 objects were selected as "other" sources: because of their positional 
instability, they are less appropriate for astrometric purposes, but are useful for providing possible ties between reference frames at other wavelengths or for other objectives. The rejection critera for the selection of the above sources were varied: the arclength formal errors $(>1$ mas), the number of observations $(<20)$, the time-span of data ( $<2$ years), the discrepancy between the cata$\operatorname{logs}(3 \sigma$ or $>0.5$ mas), the presence of an excessive source structure, or significant apparent motions.

In 1999, a first extension of the ICRF, called ICRF-Ext.1 (IERS 1999), was constructed on the basis of five years of additional observational data, spanning december 1994 through April 1999, and taking 600000 observations into account, grouped in 461 sessions. Following this extension, the list and the coordinates of the defining sources were not changed (quite normal for their definition) whereas the coordinates of the candidates and other sources were refined. In total the ICRF-Ext.1 is characterized by the addition of 59 sources to the frame, bringing the total number from 608 sources (212 "defining", 294 "candidates", and 102 "others") to 667 . In addition to the consideration of new observations, some improvements were made in the analysis models, including the tropospheric mapping function, the troposphere linear interval, the introduction of sub-diurnal EOP, and the determination of the offsets in the fixed station axis.

A second extension of the ICRF, called ICRF-Ext.2 (Fey et al. 2004), was constructed starting from both geodetic and astrometric observing programs, ranging in time from 1999 to 2002. It comes from the addition of 1.2 million observations performed during approximatively 400 sessions, and is characterized by the inclusion of 50 new sources. As was the case for the ICRF-Ext.1, the positions and errors for the defining sources remained unchanged, whereas those for the candidate and other sources were re-estimated from a denser and more accurate sample of observations. Note that ICRF-Ext. 2 received the benefits of a huge collaboration between the US Naval Observatory (USNO), the Goddard Space Flight Center (GSFC), and the National Radio Astronomy Observatory (NRAO), resulting in more than 600000 delay observations from 30 VLBA (very long baseline array) sessions in both astrometry and geodesy.

\section{Quasar catalogs at optical wavelengths}

As early as 1971 , i.e. less than 10 years after the discovery of quasars, the first catalog of these exotic objects was published. It contained 202 objects (De Veny et al. 1971). Then, various observational programs led to a latge increase in known quasars. With the exception of radio emission, which is often extended, the vast energy output of the quasars is concentrated in a very small area, which gives them a quasi ponctual aspect, thus giving birth to their name, "quasar" for "quasi stellar source". Fundamental characteristics can be found in their spectra, characterized by strong, broad, and redshifted emission lines for wavelengths in the optical and UV, together with a flat and often blue continuum from far infrared to X-ray.

Quasars' data come from a wide variety of bibliographic and electronic sources, each of them with its own resolution and energy range. X-ray observations, characterized by low resolution of the detectors necessitating an appropriate post-reduction with standard software (IRAF, MIDAS), are made from satellites, such as EXOSAT, ROSAT, ASCA, EINSTEIN. For infra-red and optical wavelengths, spectral data (i.e. determination of the flux with respect to the wavelength), can be obtained from groundbased telescopes with diameter of at least $4 \mathrm{~m}$, because of the large magnitude of the quasars. Nevertheless, ground based telescopes on the order of $1 \mathrm{~m}$ enable photometric data to be carried out, as magnitude in the usual UBVRIJHK color range. The IRAS database provides far-infrared data for quasars, as a flux for each specific band filter, whereas radio telescopes enable us to measure fluxes or brightness temperatures in specific frequency bands. Recently, several survey programs have considerably increased the number of recorded quasars.

- The first edition of the SDSS quasar catalog (Schneider et al. 2002) contains 3814 objects whose 3000 were discovered by the survey itself. To be selected as a quasar in this catalog, the extragalactic objects must have at least: one emission line with full width at a half-maximum larger than $1000 \mathrm{~km} \mathrm{~s}^{-1}$, luminosities brighter than $M_{I}=-23$, and highly reliable redshifts. The area covered by the catalog is $494 \mathrm{deg}^{2}$, which corresponds roughly to $1.2 \%$ of the sky. Their redshifts range from $z=0.15$ to $z=5$. Notice the relatively good astrometric precision of the catalog, for which the positioning accuracy of the objects is better than $0.2^{\prime \prime} \mathrm{rms}$.

- The $2 \mathrm{dF}$ QSO redshift survey (2QZ) is a huge program including approximatively 50000 quasars in the its second release that is not yet published (Croom et al. 2004). The first release contained over 10000 quasars among a total of 20590 sources listed, for which spectra were obtained using the 2-degree field facility of the Anglo-Australian Observatory. The corresponding effective area was $289.6 \mathrm{deg}^{2}$ for QSO candidates with $b_{J}$ magnitudes ranging from 18.25 to 20.85 . In the second release, the total 2QZ survey, containing 49425 quasars, covers an area of $740 \mathrm{deg}^{2}$, arranged in two $75^{\circ} \times 5^{\circ}$ declination strips centered on $\delta=-30^{\circ}$ and $\delta=0^{\circ}$. These strips extend respectively, from $\alpha=21^{\mathrm{h}} 40^{\mathrm{mn}}$ to $\alpha=3^{\mathrm{h}} 15^{\mathrm{mn}}$ and from $\alpha=9^{\mathrm{h}} 50^{\mathrm{mn}}$ to $\alpha=14^{\mathrm{h}} 50^{\mathrm{mn}}$.

- All the recorded quasars were gathered as a compilation on a regular basis by Véron-Cetty \& Véron, in particular, in ESO Scientific Reports (1984 to 2000). In the following the corresponding catalogs will be named VV. In order to identify a quasar from any other object, Véron-Cetty \& Véron (2001) established well-defined criteria: the object must be starlike or contain a starlike nucleus with broad emission lines brighter than magnitude $M_{B}=-23$. This last magnitude is estimated through a law involving the apparent magnitude $m$, the distance $D$, and corrective factors $k$ and $\Delta m(z)$ (Véron-Cetty \& Véron 2001):

$M=m+5-5 \log D-k+\Delta m(z)$

where distance $D$ is itself defined from Hubble's law. $D=$ $c / H_{0} \times A, A$ being the luminosity distance (Terrell 1977) given by:

$A=z\left[1+\frac{z\left(1-q_{0}\right)}{\sqrt{1+2 q_{0} z}+1+q_{0} z}\right]$

where $q_{0}=0$ and $z$ is the redshift.

In this equation, $k$ is dependent on the redshift in the form: $k=$ $-2.5 \log (1+z)^{1-\alpha}$, and $\Delta m$ is a correction to $k$ as determined by this last equation, taking the spectral profile of the quasars into account.

In addition to quasars, VV catalogs contain two kinds of objects: BL Lac objects and active galaxies. Recall that BL Lacertae objects (BL Lac for short) are characterized not only by absence of emission lines, but also by a strong continuum stretching from radio through X-ray frequencies. They can exhibit dramatic variability. Thus, its name persits from the original mis-identification of a prototype for such galaxies as a variable star in our own galaxy in the Lacerta constellation. Active galaxies are characterized by a small core of emission embedded in an otherwise 


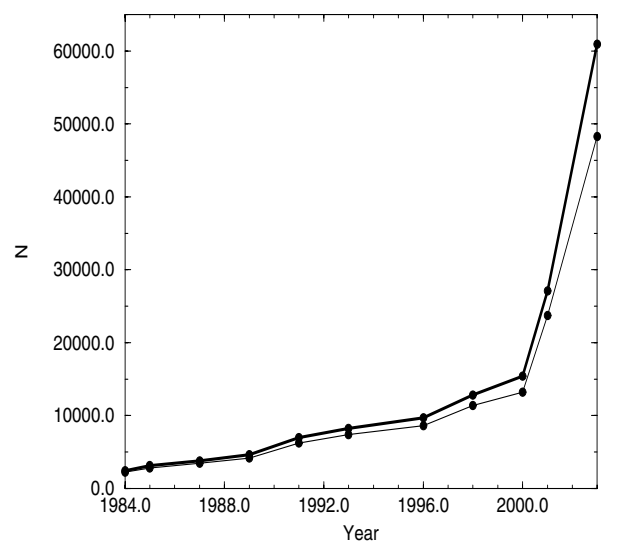

Fig. 1. Number of extra-galactic objects (bold) and quasars (normal) recorded by Véron-Cetty \& Véron from 1984 to 2003 in their publications.

typical galaxy. This core may be very bright compared to the rest of the galaxy. Among the active galaxies recorded in the VV2003 release, a large proportion (corresponding to roughly $78.2 \%$ of the sample) are Seyfert I galaxies. Recall that Seyfert I and Seyfert II galaxies have both emission spectral lines in their nuclei, as evidence of highly excited gas, but the difference between the two types arises in the shape of the emission lines, which are much broader for the first type, thus characterizing gas velocities of over $1000 \mathrm{~km} \mathrm{~s}^{-1}$.

The most extended version of the Véron-Cetty \& Véron catalog (Véron-Cetty \& Véron 2003), referred to as VV2003 in the following, adds the objects recorded in the second release of the $2 \mathrm{dF}$ quasar catalog to the earlier version, along with the first part of the SLOAN catalog. Therefore, it almost doubles the number of recorded quasars, while the number of active nuclei has also increased considerably: at total VV2003 contains 48921 quasars, 876 BL LAC objects, and 15069 active galaxies, for which a majority (11777, i.e. $78 \%$ ) are Seyfert galaxies. Like the previous edition, the VV2003 catalog includes a position at $\mathrm{J} 2000$.0, a redshift and $U B V$ photometry. When available, flux densities expressed in Jy, at $6 \mathrm{~cm}$ and $11 \mathrm{~cm}$, are given, but in fact a very small proportion of objects have been observed at radio wavelengths: only 2850 quasars recorded at $6 \mathrm{~cm}(5.8 \%)$ and only $1418(2.9 \%)$ at $11 \mathrm{~cm}$. Nearly all of these last ones (1411 objects) were observed in the two radio bandwidths.

In Figs. 1-3, we show the total number of quasars, BL Lac objects, and active galaxies recorded in all Véron Cetty \& Véron's publications, with respect to the year of publication. This number has increased dramatically in recent years, showing an exponential-like curve. This is due to the outcome of new programs, such as the $2 \mathrm{dF}$ (2 degree field) QSO Redshift Survey (Crooms et al. 2004) or the SDSS.

A first release of the $2 \mathrm{dF}$ QSO redshift survey has almost doubled the number of known quasars (Veron-Cetty \& Veron 2001). This release consisted in a catalog comprising more than 10000 QSO covering an effective area of $290 \mathrm{deg}^{2}$. Their detection was based on single spectroscopic observations at the Anglo Australian Telescope. All the objects have their magnitude $b_{j}$ included in the range $18.25<b_{j}<20.25$.

\section{Cross-identification between ICRF and catalogs of quasars}

As seen previously the present access to the ICRS at radiowavelengths is given by a set of 717 extragalactic radio sources

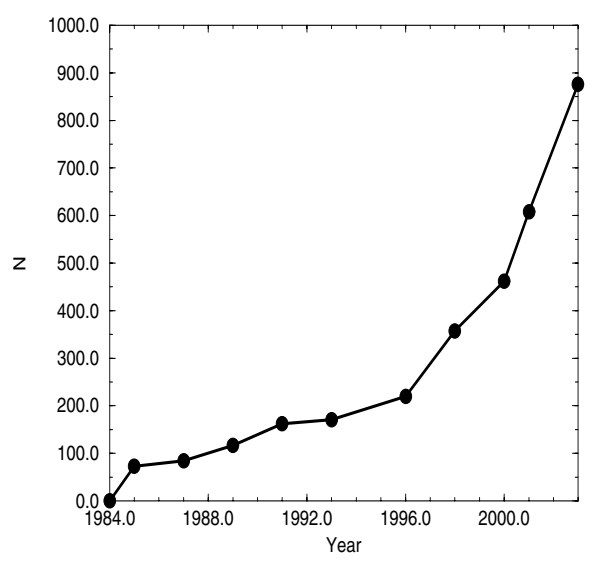

Fig. 2. Number of BL Lac objects recorded by Véron-Cetty \& Véron from 1984 to 2003 in their publications.

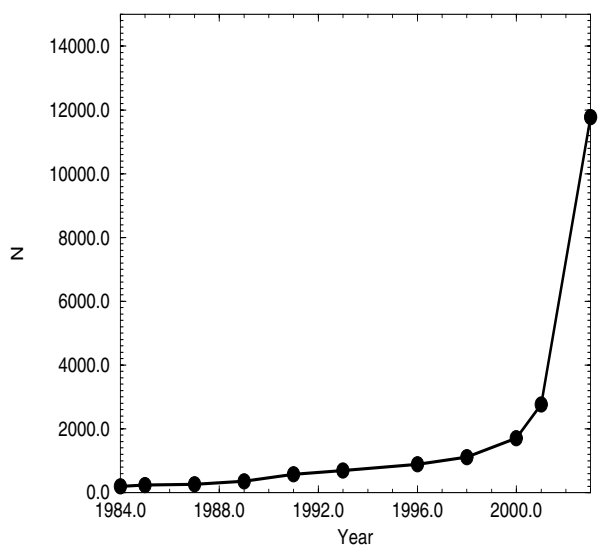

Fig. 3. Number of active galaxies recorded by Véron-Cetty \& Véron from 1984 to 2003 in their publications.

in ICRF-Ext.2 (Fey et al. 2004). In this extended version of the ICRF, four categories of sources can be considered. In addition to the "defining", the "candidate", and sources quoted as "other" present in the original ICRF version, we consider a fourth category of the additional sources present in the extensions to the catalog. Recall that the first category designates 212 sources whose coordinates are so stable that they have conventionally been fixed: their coordinates, determined at the level of the submilliarcsecond, remain unchanged with respect to the first realization of the frame (Arias et al. 1995), and they fully represent the ICRF. The second category represents 294 sources that might be chosen as defining ones because they are very stable, but they need more observations to make conclsions about their astrometric quality.

The 102 "other" sources are useful to the densification of the frame, but do not garantee the astrometric stability enough to be ranged in one of the two first categories. In addition, we consider the 109 new sources coming from the two consecutive extensions of the ICRF, and not yet distributed in any of the three above categories.

In Table 1 we indicate the number of cross-identified objects between the ICRF in its original version (without extension, that is to say containing 608 objects) and the VV2003 catalog for two different values of the threshold adopted for searching a counterpart, $4^{\prime \prime}$ and $2^{\prime \prime}$. A significant proportion of ICRF objects do not belong to the VV2003 catalog: $16.6 \%$ of the defining sources, $29.9 \%$ of the candidate and $10.8 \%$ of the other ones. Note that the VV2003 is not an astrometric catalog, which 
Table 1. Number of quasars cross-identified between the recent catalog of Véron Cetty \& Véron (2003) and the three categories of catalogs used in the construction of the ICRF: 212 defining sources, 294 candidate sources and 102 other sources. Two different angular thresholds have been chosen for the cross-identification: $2^{\prime \prime}$ and $4^{\prime \prime}$. The rms concern the difference in the positions, in arc-seconds, for the cross-identified sources.

\begin{tabular}{lrrrr}
\hline \hline Catalog & $\begin{array}{r}\text { Threshold } \\
\left({ }^{\prime \prime}\right)\end{array}$ & $\begin{array}{r}\text { Defining } \\
\text { ICRF }\end{array}$ & $\begin{array}{r}\text { Candidate } \\
\text { ICRF }\end{array}$ & $\begin{array}{r}\text { Other } \\
\text { ICRF }\end{array}$ \\
\hline Quasars & 4 & $132 / 212$ & $160 / 294$ & $69 / 102$ \\
& & $(62.2 \%)$ & $(54.4 \%)$ & $(67.6 \%)$ \\
rms (") $\alpha / \delta$ & & $0.82 / 0.74$ & $0.91 / 0.75$ & $0.69 / 0.66$ \\
BL LAC & 4 & $28 / 212$ & $24 / 294$ & $11 / 102$ \\
& & $(13.2 \%)$ & $(8.2 \%)$ & $(10.7 \%)$ \\
rms (") $\alpha / \delta$ & & $0.58 / 0.56$ & $0.88 / 0.55$ & $0.86 / 0.55$ \\
Active galaxies & 4 & $17 / 212$ & $22 / 294$ & $11 / 102$ \\
& & $(8.0 \%)$ & $(7.5 \%)$ & $(10.7 \%)$ \\
rms (") $\alpha / \delta$ & & $0.79 / 0.66$ & $0.93 / 0.56$ & $0.96 / 0.74$ \\
All objects & 4 & $177 / 212$ & $206 / 294$ & $91 / 102$ \\
rms (") $\alpha / \delta$ & & $(83.4 \%)$ & $(70.1 \%)$ & $(89.2 \%)$ \\
\hline Quasars & 2 & $128 / 212$ & $152 / 294$ & $69 / 102$ \\
& & $(60.4 \%)$ & $(51.7 \%)$ & $(67.6 \%)$ \\
rms (") $\alpha / \delta$ & & $0.74 / 0.65$ & $0.84 / 0.68$ & $0.69 / 0.66$ \\
BL LAC & 2 & $28 / 212$ & $24 / 294$ & $11 / 102$ \\
& & $(13.2 \%)$ & $(8.2 \%)$ & $(10.7 \%)$ \\
rms (") $\alpha / \delta$ & & $0.58 / 0.56$ & $0.88 / 0.55$ & $0.86 / 0.55$ \\
Active galaxies & 2 & $17 / 212$ & $21 / 294$ & $10 / 102$ \\
& $(8.0 \%)$ & $(7.1 \%)$ & $(9.8 \%)$ \\
rms (") $\alpha / \delta$ & & $0.79 / 0.66$ & $0.85 / 0.67$ & $0.59 / 0.71$ \\
All objects & 2 & $173 / 212$ & $205 / 294$ & $90 / 102$ \\
rms (") $\alpha / \delta$ & $(81.6 \%)$ & $(67.0 \%)$ & $(88.2 \%)$ \\
\hline
\end{tabular}

explains why reducing the identification threshold from $4^{\prime \prime}$ to $2^{\prime \prime}$ leads to the loss of 14 cross-identifications in total (4 and 8 quasars of the defining and candidate sources, respectively, and 1 active galaxy of each of the candidates and of the other sources).

In Table 2 we represent the results of the cross-identifications between the whole original ICRF catalog (608 sources) and its extended version (ICRF-Ext.2). The overall percentage of crossidentified sources is not improved by the extension, as its value decreases from $77.9 \%$ from the original ICRF to $75.0 \%$ with the 4 " threshold and from $75.7 \%$ to $72.5 \%$ with the 2 " threshold.

From this study we can conclude that, although the ICRF is the primary and by far the more accurate catalog for astronomers in an astrometric sense, basic information is lacking for a significant proportion of the objects contained in the catalog, such as the $V$ photometry and the redshift estimations. Nevertheless, thanks to the cross-identified objects above, it is possible to compare the general characteristics of a majority of ICRF quasars (redshifts, magnitudes, radio flux) with respect to all the quasars belonging to the VV2003 catalog, as follows.

\section{Photometric properties of the ICRF quasars}

In Figs. 4 and 5, we present first the histograms of the $V$ magnitudes of the 48921 quasars recorded in the VV2003 catalog then those of the 390 cross-identified sources of the ICRF-Ext. 2 (within the $2^{\prime \prime}$ identification threshold).

A large majority of these last ones (roughly 75\%) are included in the range $16.5<V<19.5$, whereas the same percentage of the VV2003 objects concerns the range $18.5<V<21.0$. From that we can assert that ICRF selected objects are generally bright objects in optical wavelengths compared to the average. From a comparison of the two histograms we can add that the
Table 2. Number of quasars cross-identified between the recent catalog of Véron Cetty \& Véron (2003) and two categories of catalogs used in the construction of the ICRF: the combination of the defining, candidate and other sources of Table 1, with 608 sources, and its extension, the ICRF-Ext. 2 catalog containing 717 sources. Two different angular thresholds have been chosen for the cross-identification: $2^{\prime \prime}$ and $4^{\prime \prime}$. The rms concern the differences in the positions, in arcsecond, for the cross-identified sources.

\begin{tabular}{lrrr}
\hline \hline Catalog & $\begin{array}{r}\text { Threshold } \\
\left({ }^{\prime \prime}\right)\end{array}$ & $\begin{array}{r}\text { ICRF } \\
(3 \text { categories })\end{array}$ & ICRF-Ext.2 \\
VV2003 & 4 & $361 / 608$ & $403 / 717$ \\
Quasars & & $(59.4 \%)$ & $(56.2 \%)$ \\
& & $0.84 / 0.73$ & $0.84 / 0.72$ \\
rms (') $\alpha / \delta$ & 4 & $63 / 608$ & $66 / 717$ \\
BL LAC & & $(10.4 \%)$ & $(9.2 \%)$ \\
& & $0.76 / 0.55$ & $0.77 / 0.55$ \\
rms (") $\alpha / \delta$ & 4 & $50 / 608$ & $69 / 717$ \\
Active galaxies & & $(8.2 \%)$ & $(9.6 \%)$ \\
& & $0.90 / 0.64$ & $0.93 / 0.82$ \\
rms (") $\alpha / \delta$ & 4 & $474 / 608$ & $538 / 717$ \\
All objects & & $(77.9 \%)$ & $(75.0 \%)$ \\
rms (") $\alpha / \delta$ & 2 & $349 / 608$ & $390 / 717$ \\
Quasars & & $(57.4 \%)$ & $(5.4 \%)$ \\
& & $0.77 / 0.67$ & $0.77 / 0.65$ \\
rms (") $\alpha / \delta$ & & $63 / 608$ & $66 / 717$ \\
BL LAC & 2 & $(10.4 \%)$ & $(9.2 \%)$ \\
& & $0.76 / 0.55$ & $0.77 / 0.55$ \\
rms (") $\alpha / \delta$ & & $48 / 608$ & $64 / 717$ \\
Active galaxies & 2 & $(7.8 \%)$ & $(8.9 \%)$ \\
& & $0.78 / 0.64$ & $0.75 / 0.67$ \\
rms (") $\alpha / \delta$ & & $460 / 608$ & $520 / 717$ \\
All objects & & $(75.7 \%)$ & $(72.5 \%)$ \\
rms (") $\alpha / \delta$ & & & \\
\hline
\end{tabular}

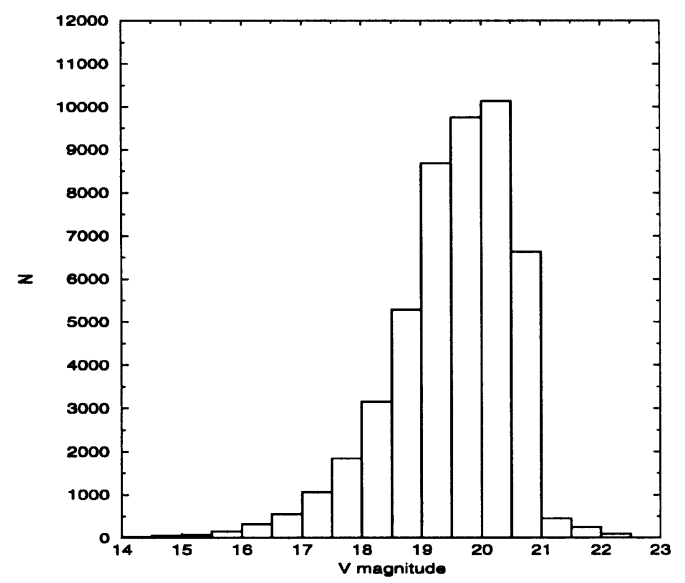

Fig. 4. Histogram showing the number of quasars of VV2003 catalog with respect to their $V$-magnitude.

proportion of quasars among the brightest ones $(15<V<17)$ chosen to build up the ICRF is comparatively much higher than in the VV2003 catalog. Nevertheless, at the other extrem of the histogram, the presence of very faint ICRF sources, with $V>20$, indicates that their small flux at optical wavelengths does not seem to be a handicap for their astrometric quality, which essentially depends on the amplitude and the stability of their emission at radio wavelengths.

In Figs. 6 and 7, we show the results of the same kind of study as above, but at the $6 \mathrm{~cm}$ wavelength, which is slightly different from that of the $S$ band $(3.5 \mathrm{~cm})$, which is one of the two bands generally used for VLBI observations. We observe 


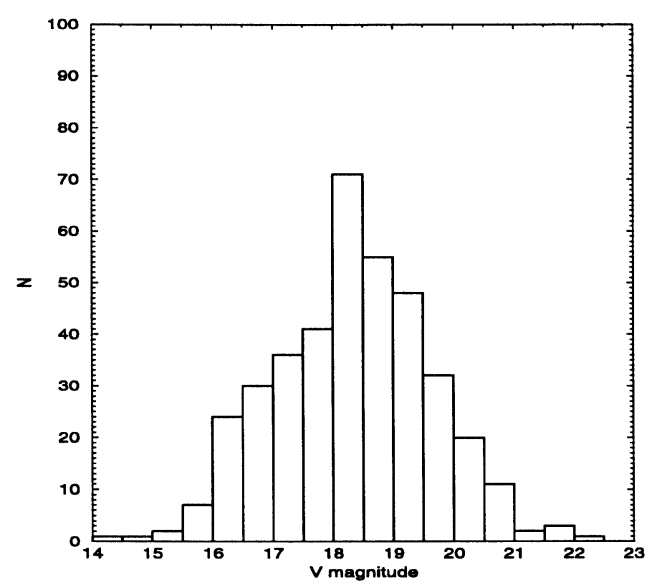

Fig. 5. Histogram showing the number of quasars of the ICRF-Ext. 2 catalog cross-identified with the VV2003 catalog, with respect to their $V$-magnitude.

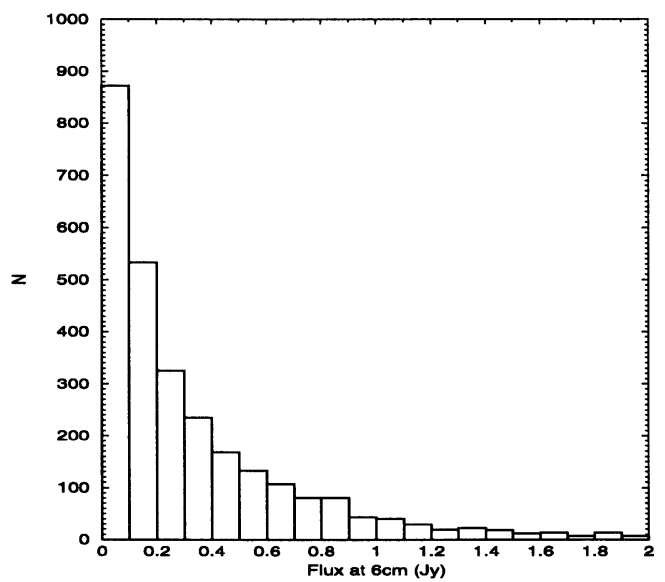

Fig. 6. Histogram showing the number of quasars in the VV2003 cata$\log$ with respect to their radio flux at $6 \mathrm{~cm}$.

that for both catalogs most of the sources are located in a flux span from 0 to $2 \mathrm{Jy}$, but with a very different distribution for the two catalogs: while the number of sources is steadily decreasing with respect to the flux at $6 \mathrm{~cm}$ in the VV2003 catalog, the selected cross-identified ICRF sources were chosen in a large proportion (roughly $80 \%$ ) in the range between $0.3 \mathrm{Jy}$ and $1.2 \mathrm{Jy}$, for which the distribution is quite regular, with a peak between 0.8 and $0.9 \mathrm{Jy}$. Thus, they are by far much brighter in average than the sources in VV2003.

Figures 8 and 9 show the results of the same study but concerning the flux at $11 \mathrm{~cm}$ (close to the VLBI X band at $13 \mathrm{~cm}$ ). The distribution of the sources for both catalogs is a little more extended than in the previous case, ranging between 0 and $5 \mathrm{Jy}$. Still, a difference appears in the general feature of the histograms from the VV2003 catalog (Fig. 8) and from the ICRF-Ext.2 cata$\log$ (Fig. 9). While we observe a very sharp increase up to $0.3 \mathrm{Jy}$, and then a regular decrease in the number of sources with respect to the flux at $11 \mathrm{~cm}$ in the first case, the distribution of the number of sources looks quite stable between 0.5 and $1.5 \mathrm{Jy}$. in the second one. Still here we see that the ICRF flux are more luminous in average but the contrast is less than at $6 \mathrm{~cm}$ (Figs. 6 and 7).

From the comparison of the histogram profiles for redshifts, both for the 48920 quasars of the VV2003 catalog (Fig. 10) and the sources of the ICRF-Ext.2 (Fig. 11), we can observe

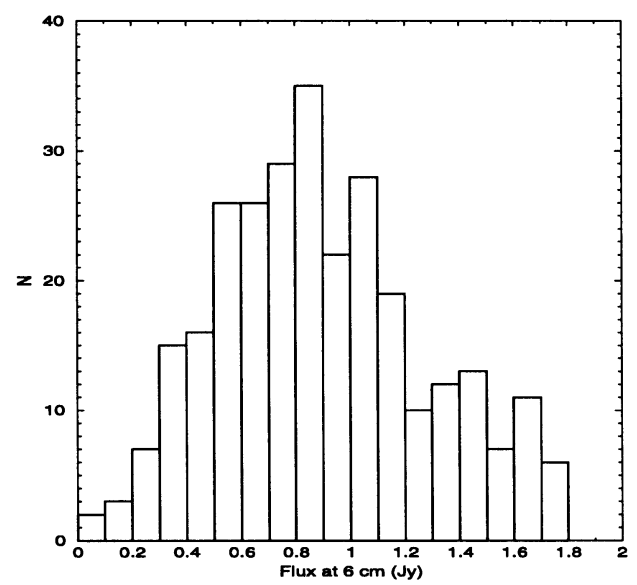

Fig. 7. Histogram showing the number of defining sources in the ICRF cross-identified with VV2003, with respect to their flux at $6 \mathrm{~cm}$.

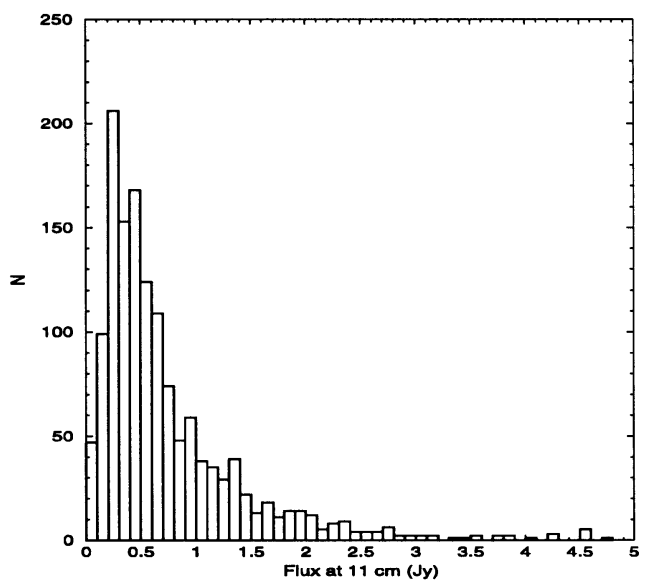

Fig. 8. Histogram showing the number of quasars in the VV2003 cata$\log$ with respect to their radio flux at $11 \mathrm{~cm}$.

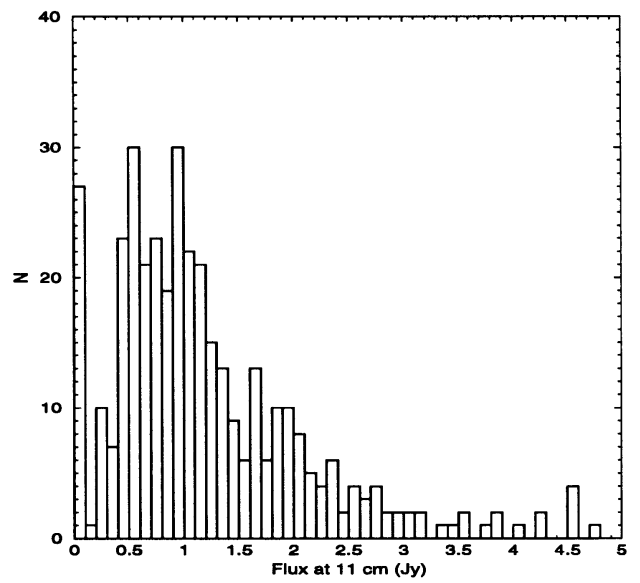

Fig. 9. Histogram showing the number of defining sources in the ICRF cross-identified with VCV2003, with respect to their flux at $11 \mathrm{~cm}$.

that the sources of the ICRF have been chosen generally among relatively close objects, the majority of which have a value of $z<1.5$, which corresponds to the median line, which means the number of recorded quasars is roughly the same after and before that line. Notice also, as a counterpart, the relative amount of very distant objects with redshifts higher than $z=3$ in the ICRF-Ext. 2 catalog. 


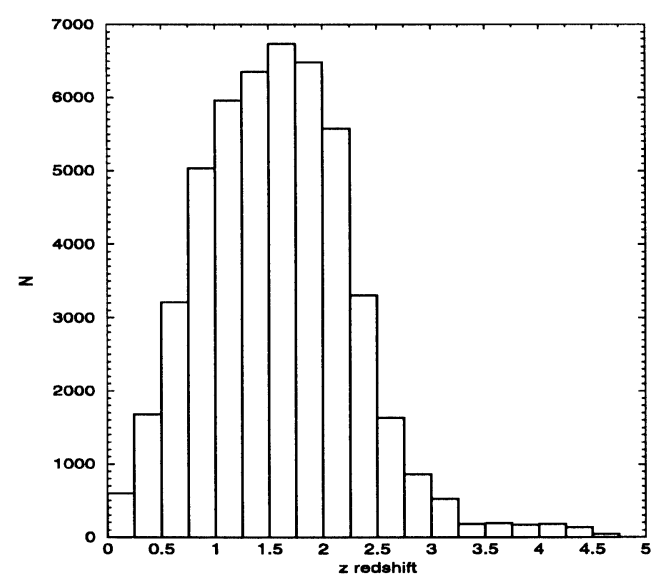

Fig. 10. Histogram showing the number of quasars in the VV2003 catalog, with respect to their redshift.

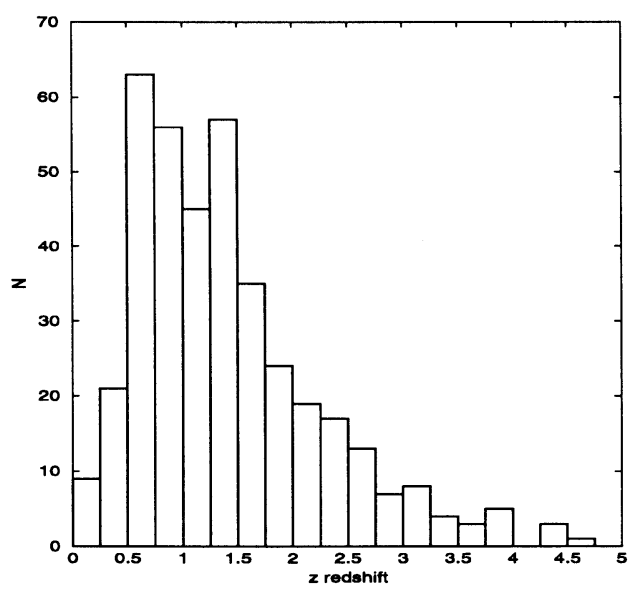

Fig. 11. Histogram showing the number of quasars in the ICRF-Ext.2 catalog cross-identified with the VV2003 catalog, with respect to their redshift.

As the redshifts of quasars are directly related to their distance by the intermediary of Hubble's law, it is interesting to plot the $V$-magnitude of these objects, which to some extent should also depend on the distance with respect to their redshift. This is properly done in Fig. 12, both for the VV2003 recorded objects and for the cross-identified ICRS quasars. In the left part of the figure, we can observe that for a given redshift, there is well-defined threshold for the upper limit in $V$. This means that for a given redshift, i.e. at a given distance, it is not possible to find a quasar that is fainter than this magnitude threshold. As a consequence, a very well-defined limiting curve can be described above which no quasar can be found (in fact we can detect the presence of 3 quasars in this forbidden zone). Even if the link is not explicit, the threshold above is undoubtedly implicite in formula (1), which presents the relationships between the absolute and apparent magnitude of an object as a function of its redshift, through distance $D$, together with the condition $M_{B}<-23$, for considering an object as a quasar. Notice also the presence of several quasars of the ICRF-Ext.2 catalog located very close to the threshold above, which means that they should not have been considered as a quasar in the VV2003 catalog, if their magnitude were slightly larger. Finally, notice the lack of completeness starting from $V=21$, characterized by a horizontal limit upon which a relatively very small proportion of ICRF, as well as VV2003 quasars, have been identified.

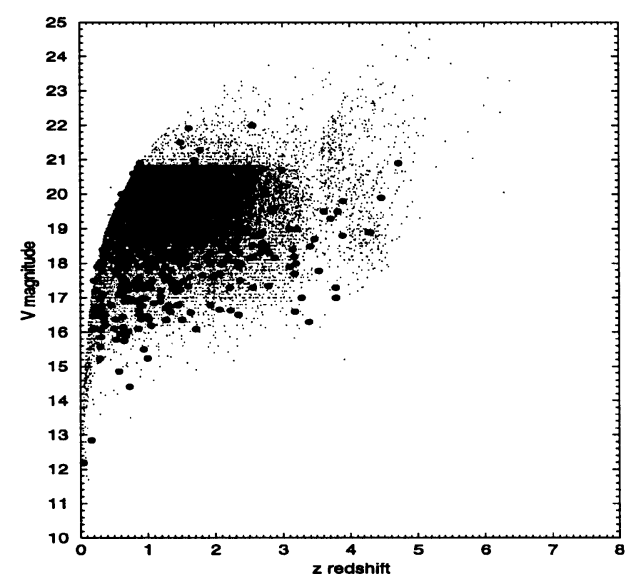

Fig. 12. Curve showing the $V$-magnitude of quasars in the VV2003 catalog (dots) and of the ICRF defining sources cross-identified with this last catalog (large full circles), with respect to their redshift $z$.

\section{About the sky coverage of the ICRF quasars and other catalogs}

The ICRF has been adopted as the primary conventional catalog since 1998. Nevertheless, despite its very high astrometric quality, its sky coverage is fairly poor, which is obviously due to the small number of objects: 212 if we take only the defining sources into account, 608 when also considering the candidate and other sources, 717 if we include the whole sample of the ICRF-Ext.2 catalog. Indeed, the primary realization of the ICRS at optical wavelengths was carried out using the position of roughly 100000 stars of the Hipparcos catalog, and at a larger and less accurate extent using the 3 millions objects of the Tycho-2 catalog (Hog et al. 2000).

We focused our study on the intrinsic sky coverage of the ICRF and other quasar catalogs, measured in the following way. We selected points on the celestial sphere separated by equal intervals, that is to say, one degree in right ascension $\alpha$ and also in declination $\delta$. Then for each of the points selected, we calculated the closest object of the selected catalog we studied. We payed attention to the fact that the points are not regularly located on the sphere because of the $\cos \delta$ factor, but we chose this distribution for sake of simplicity.

Figure 13 uses bi-dimensional coordinates $(\alpha, \delta)$ to represent the zones of the celestial sphere in black, for which the closest distance to a defining source of the ICRF (212 objects at all) is greater than $10^{\circ}$ (on the opposite side, the white zones correspond to points for which the closest ICRF quasar is at an angular distance less than $10^{\circ}$ ). The reader should be aware of distortion due to the $\cos \delta$ factor at the high declinations, for which one degree separating two points in right ascension correspond to a very small angular distance. The same study is done in Fig. 14 but by replacing the defining sources by the 717 extragalactic sources of the ICRF-Ext.2. We note that the depletion zones have decreased considerably, since limited to small areas. Obviously, the two figures show that the sky coverage is more deficient in the southern hemisphere than in the northern one, for wich the zones of depletion covers a considerably smaller surface.

In Fig. 15 we present the same kind of study as above, but with the 48290 quasars of the VV2003 catalog and a considerably smaller angular distance $\left(1^{\circ}\right)$. A significant part of the sky, corresponding to the white zone, is covered by the recorded quasars at this level of angular distance, except two depletion 


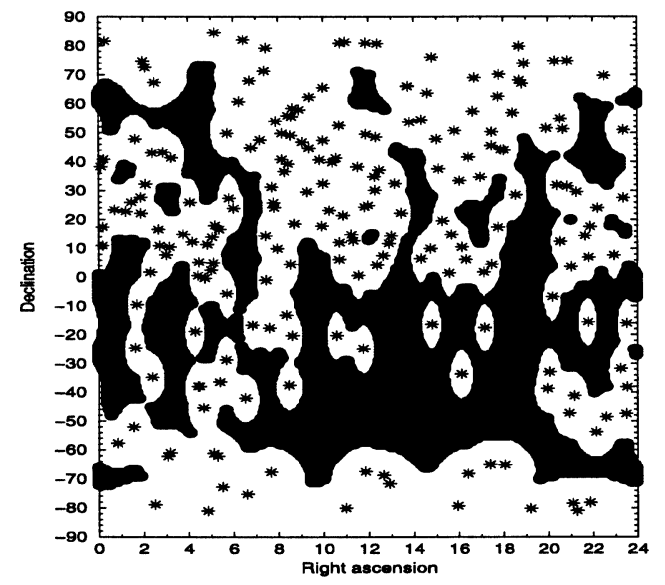

Fig. 13. Plot in black of the zones of the sky for which the shortest distance to a defining source of the ICRF catalog (212 sources in all) is greater than $10^{\circ}$. Stars represent the positions of the defining sources.

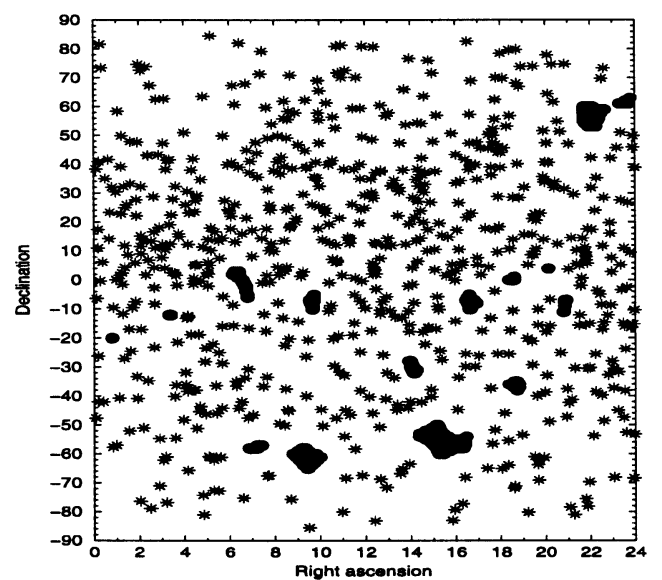

Fig. 14. Plot in black of the zones of the sky for which the shortest distance to a source of the ICRF.Ext-2 catalog ( 717 sources in all) is greater than $10^{\circ}$. Stars represent the positions of the selected set of sources.

zones covering the intervals roughly from $5^{\mathrm{h}}$ and $8^{\mathrm{h}}$ and from $17^{\mathrm{h}}$ to $20^{\mathrm{h}}$. These two zones more or less correspond to the presence of the galactic plane (the Milky Way) through which no extragalactic object can be easily observed. Still, here we must keep in mind that the large black zone close to the southern hemisphere in fact covers a very small area of the sky. Nevertheless, such a large depletion zone is not present around the north pole.

\section{Conclusion}

In this paper we have cross-identified the ICRF, constructed starting from observations at radio wavelengths with the VV2003 (Véron-Cetty \& Véron 2003) compilation catalog, which is the denser catalog of quasars presently available, at optical wavelengths. We have shown that a significant part (17\% of the defining, $30 \%$ of the candidate, and $11 \%$ of the other sources) of the ICRF sources are not recorded in VV2003. Nevertheless, the remaining common identifications enabled us to point out some general trends of the ICRF quasars with respect to the general properties of quasars (magnitude, redshift, and flux at $6 \mathrm{~cm}$ and $11 \mathrm{~cm}$ ) established statistically from the large set (48 290 objects) of the VV2003 catalog. They are generally

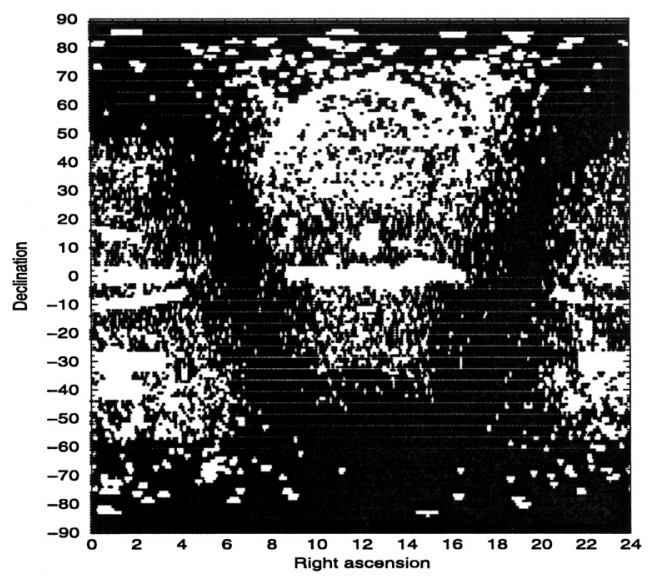

Fig. 15. Plot in black of the zones of the sky for which the shortest distance to a quasar of the VV2003 catalog (48 520 sources at all) is larger than $1^{\circ}$.

much brighter, much closer, and their flux at radio wavelengths $(6 \mathrm{~cm}$ and $11 \mathrm{~cm})$ more dispersed, as in this last catalog. In addition of the 403 quasars belonging both to the ICRF-Ext. 2 catalog and to the VV2003 catalog, 63 BL LAC objects and 69 active galaxies were cross-identified.

In the second part we compared the sky coverage of the ICRF catalog and of the VV2003. While in the first case some zones of the sky are not covered at the angular distance of $10^{\circ}$, in the second case a large part of the sky is close to a quasar at an angular distance of $1^{\circ}$. Nevertheless, taking the 717 sources of the ICRF-Ext. 2 catalog into account nearly cancels the depletion zones occupying roughly half of the sky when only the 212 defining ICRF sources are chosen.

There is no doubt that, with the increasing number of quasars detected at an exponential level, it will be possible to drastically improve the astrometric measurements for the whole sky in the near future. Of course the up-coming of the GAIA space mission will be able to detect around 500000 quasars, which corresponds roughly to 12 quasars/square degree. In comparison, the present population of the VV2003 catalog, that is to say, 48290 objects, corresponds to a mean coverage of 1.17 quasar/square degree whereas the ICRF Ext.2 coverage correponds to 0.017 quasar/square degree. Therefore this paper might be a useful initial work to when studying how to extend the ICRF in the near future both in astrometric and photometric stage.

\section{References}

Arias, E. F., Charlot, P., Feissel, M., \& Lestrade, J. F. 1995, A\&A, 303, 604 Crooms, S. M., Smith, R. J., Boyle, B. J., et al. 2001, MNRAS, 322, L29 De Veny, J. B., Osburn, W. M., \& James, K. 1971, PASP, 83, 611 Fey, A. L., Ma, C., Arias, E. F. et al. 2004, AJ, 127, 3587 Fricke, W., Schwan, H., Lederle, T., et al. 1988, Fifth Fundamental catalog (FK5). Part I: the Basic Fundamental Stars (Verlag Braun: Karlsruhe)

Hog, E., Fabricius, C., Makarov, V. V., et al. 2000, A\&A, 335, L27

IAU 2000, in Proceedings of the Twenty-fourth General Assembly -Manchester 2000, resolution B1.2, 39

IERS Annual Report 1999, edn. Obs. Paris

Kovalevsky, J. 1998, Highlights of Astronomy, Vol. 11A, XXIIIrd General Assembly of the IAU, 1997 (Kluwer Academic Publ.), 536

Ma, C., Arias, E. F., Eubanks, T. M., et al. 1998, AJ, 116, 516

Richards, G. T., Fan, X., Schneider, D. P., et al. 2001, AJ, 121, 2308

Terrell, J. 1977, Am. J. Phys., 45, 869

Véron-Cetty, M. P., \& Véron, P. 2001, A\&A, 374, 92

Véron-Cetty, M. P., \& Véron, P. 2003, A\&A, 412, 399 